\title{
Using Advanced Microscopy to Promote Science Education in an After School Enrichment Program
}

\author{
Guichuan Hou and Chishimba N. Mowa
}

Department of Biology and Dewel Microscopy Facility, Appalachian State University, Boone, NC 28608-2027

Advanced microscopies, such as transmission and scanning electron microscopy (TEM, SEM), video and confocal microscopy are essential research tools in many scientific disciplines. Here, we describe the systematic use of these complex technologies as teaching tools to build selfefficacy in high school students, excite their interest and critical thinking in sciences, and inspire students in pursuing careers in science. Appalachian Student Experience-based EDucation (Appal-SEED) Academy at Appalachian State University (ASU) is in its third year through support by Burroughs Welcome Fund. The Academy primarily serves minority, low income and home schooled high school students in our region who traditionally have less access to scientific expertise and cutting-edge technology. As a part of the Appal-SEED Academy, which has four different disciplines, the Mountain TOP scope program is designed for students to learn more about microscopes, including how different microscopes serve scientists in different disciplines. During the first year of the program, students explored the stereo, compound, and scanning electron microscopes (SEM) and learned the basics of digital imaging and image analysis by examining a variety of samples. Students also explored the new-found microscopic world around them, including intermingled filaments (fungal hyphae) on their backyard trees and various interesting lives in the pond water. The first-year students graduated at the end of 2008-2009 academic year. In the second year of the program, new students were recruited and the new group of students explored optical microscopes and SEM. In addition, they used laser scanning confocal microscopy and learned its various applications using a variety of samples, such as ferns and forensic samples. This group of students successfully graduated at the end of 20092010 academic year. Currently, we are in the third year of the program. This cohort of students have been introduced to our new scanning transmission electron microscope (supported by NSF MRI-0922697) to examine many microorganisms and sub-cellular structures, such as rotavirus and mitochondria. In conclusion, the Mountain TOP scope program has been able to achieve its primary goal, namely to improve the students' self-efficacy, to have fun experience in science by exposing them systematically to cutting-edge modern microscopy, and to increase their chance in pursuing science-related careers in future. The Appal-SEED Academy is supported by Burroughs Wellcome Fund and ASU. The Mountain TOP scope program is conducted in the Dewel Microscopy Facility at ASU. 